\title{
Zur Theorie der thermoelektrischen Effekte in Halbleitern
}

\author{
Von Otfried Madelung \\ Aus dem Forschungslaboratorium der Siemens-Schuckert-Werke AG., Erlangen \\ (Z. Naturforschg. 13 a, 22-25 [1958] ; eingegangen am 9. November 1957)
}

Die Grundlagen der Theorie der thermoelektrischen Effekte in Halbleitern werden diskutiert. Dabei wird auf einige in der Literatur bestehende Unklarheiten in der Definition der Energiestromdichte und der in der Boltzmansschen Stationaritätsbedingung auftretenden Kraft $m \dot{\mathfrak{v}}$ hingewiesen.

Die Leitungsprozesse in einem Festkörper unter dem Einfluß angelegter Felder und Temperaturgradienten sind schon seit langem Gegenstand zahlreicher Untersuchungen gewesen. Die Theorie dieser Prozesse geht aus von der Annahme, daß der Körper einteilbar ist in makroskopisch kleine Bereiche, in denen lokales Gleichgewicht herrscht, denen also eine definierte Temperatur und ein elektrochemisches Potential zugeordnet werden kann. In diesen Bereichen gelten die bekannten thermodynamischen Relationen zwischen Entropie, innerer Energie, Teilchenzahl usw. Die Thermodynamik irreversibler Prozesse liefert dann, ausgehend von diesen Grundvorstellungen, Beziehungen zwischen den Teilchen- und Energiestromdichten und den treibenden Kräften des elektrochemischen Potentials und des Temperaturgradienten mit Koeffizienten, die unter bestimmten Modellvorstellungen berechnet werden können.

Bei der Ableitung der thermoelektrischen Effekte aus diesen Beziehungen sind nun in der Literatur eine Reihe von Vernachlässigungen vorgenommen worden, die es zweckmäßig erscheinen lassen, die Grundgedanken der Theorie nochmals zusammenzustellen und kritisch zu diskutieren.

\section{Thermodynamische Grundlagen, Definition der Energiestromdichten}

Wir gehen aus von der Gleichung

$$
T \mathrm{~d} s=\mathrm{d} u+p \mathrm{~d} v-\sum_{i} \zeta_{i} \mathrm{~d} n_{i},
$$

wo $s, u, v, n$ die Entropie, innere Energie, das Volumen und die Teilchenzahl (jeweils bezogen auf die Volumeneinheit), $T$ die Temperatur und $\zeta_{i}$ das elektrochemische Potential der $i$-ten Teilchensorte bedeuten. Vernachlässigen wir Volumenänderungen und

1 vgl. etwa die Diskussion bei A. Sommerfeld, Vorlesungen über theoretische Physik, Bd. V, § 21, Dieterichsche Verlagsbuchhandlung, Wiesbaden 1952. beschränken wir uns auf eine Teilchensorte (die Erweiterung auf mehrere Teilchensorten bereitet keine Schwierigkeiten), so läßt sich (1) in folgender Form schreiben:

$$
T \frac{\partial s}{\partial t}=\frac{\partial u}{\partial t}-\zeta \frac{\partial n}{\partial t} .
$$

Unter Einführung einer Entropiestromdichte $\boldsymbol{\sigma}$, einer Energiestromdichte $\mathfrak{m}$ und einer Teilchenstromdichte j erhält man dann mit Benutzung der Kontinuitätsgleichungen

$$
\begin{gathered}
\frac{\partial u}{\partial t}+\operatorname{div} \mathfrak{m}=0, \quad \frac{\partial n}{\partial t}+\operatorname{div} \mathfrak{j}=0, \\
T \frac{\partial s}{\partial t}=-\operatorname{div} \mathfrak{m}+\zeta \operatorname{div} \mathfrak{j}
\end{gathered}
$$$$
\text { ode } \frac{\partial s}{\partial t}+\operatorname{div} \sigma=\mathfrak{m} \cdot \operatorname{grad} \frac{1}{T}-\mathrm{j} \cdot \operatorname{grad} \frac{\zeta}{T} .
$$

Hier bedeutet das zweite Glied links die Divergenz der Entropiestromdichte $\boldsymbol{\sigma}=1 / T(\mathfrak{m}-\zeta \mathfrak{j})$, während die rechte Seite die lokale Entropieerzeugung ist $^{1}$. Da die rechte Seite von (5) eine lineare Funktion in den "Flüssen " $\mathfrak{w}$ und $-j$ ist, können wir diese als lineare Funktionen ihrer „Kräfte“ $\operatorname{grad}(1 / T)$ und $\operatorname{grad}(\zeta / T)$ ausdrücken:

$$
\begin{array}{r}
-\mathfrak{j}=L_{11} \operatorname{grad} \frac{\zeta}{T}+L_{12} \operatorname{grad} \frac{1}{T}, \\
\mathfrak{w}=L_{21} \operatorname{grad} \frac{\zeta}{T}+L_{22} \operatorname{grad} \frac{1}{T} .
\end{array}
$$

Eine Anwendung der Onsagerschen Beziehungen auf diese Gleichung ergibt schließlich noch $L_{12}=L_{21}$.

Gl. (6) stellt den Ausgangspunkt zur Berechnung der Leitungsprozesse dar. Bei der Anwendung dieser Gleichung ist jedoch zu berücksichtigen, daß die in (5) und (6) vorgenommene Aufteilung in "Flüsse“ und „Kräfte“ nicht eindeutig ist ${ }^{2,3}$. Um die verschiedenen Möglichkeiten zu diskutieren, müssen wir

2 S. R. De Groot, Thermodynamics of Irreversible Processes, North-Holland Publishing Company, Amsterdam 1951.

3 H. B. Callen, Phys. Rev. 73, 1349 [1948]. 
uns zunächst über den Begriff der Energie eines Elektrons in einem Festkörper klar werden. Dabei beziehen wir uns auf die Verhältnisse in einem Halbleiter. Führt man neben der Energie $E$ des Elektrons noch in das Energiediagramm des Halbleiters die drei charakteristischen Energieparameter $-e \psi$ (elektrostatisches Potential), $\zeta$ (elektrochemisches Potential) und $E_{\mathrm{L}}$ (Energie der unteren Kante des Leitungsbandes) ein, so läßt sich $E$ aufteilen in:

$E=-e \psi+E_{0}$ (Abspaltung des elektrostatischen Anteils),

$E=\quad \zeta+E_{\zeta}$ (Abspaltung des elektrochemischen Anteils),

$E=E_{\mathrm{L}}+E_{\mathrm{n}}$ (Aufteilung in ,potentielle“ Energie $E_{\mathrm{L}}$ und "kinetische" Energie $\left.E_{\mathrm{n}}=m v^{2} / 2\right)$.

Für die verwendeten Bezeichnungen vgl. Abb. 1 .

$\mathrm{Zu}$ diesen drei neu eingeführten charakteristischen Energien $E_{0}, E_{\zeta}$ und $E_{n}$ lassen sich nun weitere Energiestromdichten $\mathfrak{w}_{0}, \mathfrak{w}_{\zeta}$ und $\mathfrak{w}_{\mathrm{n}}$ definieren, die mit der gesamten Energiestromdichte $\mathfrak{w}$ offensichtlich in folgender Weise zusammenhängen:

$$
\begin{gathered}
\mathfrak{w}_{0}=\mathfrak{w}-(-e \psi) \mathfrak{j}, \quad \mathfrak{w}_{\zeta}=\mathfrak{w}-\zeta \mathfrak{j}, \\
\mathfrak{w}_{\mathrm{n}}=\mathfrak{w}-E_{\mathrm{L}} \mathfrak{j} .
\end{gathered}
$$



Abb. 1. Darstellung der in einem Halbleiter wichtigen Energien und Energiedifferenzen.

Die Entropiestromdichte wird dann mit diesen neuen Definitionen:

$\boldsymbol{\sigma}=\frac{1}{T}(\mathfrak{w}-\zeta \mathfrak{j})=\frac{1}{T}\left(\mathfrak{w}_{0}-\zeta_{0} \mathfrak{j}\right)=\frac{1}{T}\left(\mathfrak{m}_{\mathrm{n}}-\zeta_{\mathrm{n}} \mathfrak{j}\right) ;$

$\mathfrak{w}_{\mathrm{n}}$ ist nach Definition die Stromdichte der kinetischen Energie der Elektronen, während $\mathfrak{w}_{\zeta}$ wegen der Analogie der Beziehungen $\mathfrak{w}_{\zeta}=\sigma T$ und $q=s T$ als Wärmestromdichte bezeichnet werden kann ${ }^{3}$.

Die Kontinuitätsgleichung (3) wird dann (mit $-e \dot{\mathrm{i}}=\dot{\mathrm{i}}=$ elektr. Stromdichte, $-\operatorname{grad} \psi=E=$ elektr. Feldstärke und mit $\operatorname{div} j=0$ ) :

$$
\begin{aligned}
\frac{\partial u}{\partial t} & =-\operatorname{div} \mathfrak{m} \\
& =-\operatorname{div} \mathfrak{m}_{0}+e j \cdot \operatorname{grad} \psi=-\operatorname{div} \mathfrak{w}_{0}+\mathrm{i} \cdot(\xi) \\
& =-\operatorname{div} \mathfrak{w}_{\zeta}-\mathrm{i} \cdot \operatorname{grad} E_{\mathrm{L}}=-\operatorname{div} \mathfrak{w}_{\zeta}+\mathrm{i} \cdot\left(\xi+\mathrm{i} \cdot \operatorname{grad} \frac{\zeta_{0}}{\mathrm{e}}\right. \\
& =-\operatorname{div} \mathfrak{m}_{\mathrm{n}}-\mathrm{j} \cdot \operatorname{grad} \zeta=-\operatorname{div} \mathfrak{w}_{\mathrm{n}}+\mathrm{i} \cdot\left(\xi+\mathrm{i} \cdot \operatorname{grad} \frac{E_{\mathrm{L}_{0}}}{\mathrm{e}} .\right.
\end{aligned}
$$

Nimmt man schließlich statt $\mathfrak{w}$ als „Fluß“ in (5) und (6) eine der drei neuen Energiestromdichten, so erhält man als „Kräfte“:

Flüsse

$$
\begin{array}{r}
\text { Kräfte } \\
\operatorname{grad} \frac{\zeta}{T}, \operatorname{grad} \frac{1}{T} \\
\frac{e}{T}\left(\xi+T \operatorname{grad} \frac{\zeta_{0}}{e T}\right), \operatorname{grad} \frac{1}{T} \\
\frac{1}{T} \operatorname{grad} \zeta, \operatorname{grad} \frac{1}{T}
\end{array}
$$$$
-\mathrm{j}, \mathfrak{w}
$$$$
-\mathrm{j}, \mathrm{m}_{0}
$$$$
-\mathrm{i}, \mathrm{m}
$$$$
-\mathrm{j}, \mathfrak{m}_{\mathrm{n}} ; \frac{e}{T}\left(\xi+T \operatorname{grad} \frac{\zeta_{\mathrm{n}}}{e T}+\operatorname{grad} \frac{E_{\mathrm{Lo}}}{e}\right), \operatorname{grad} \frac{1}{T}
$$

Aus jeder dieser Kombinationen läßt sich dann ein lineares Gleichungssystem der Form (6) mit (wegen der Onsager-Beziehungen) symmetrischer Koeffizientenmatrix aufstellen. Die aus diesen verschiedenen
Möglichkeiten folgenden physikalischen Aussagen sind natürlich identisch. Man muß nur beachten, daß zum System (13), (14), (15) bzw. (16) die Kontinuitätsgleichung (9), (10), (11) bzw. (12) gehört. Dies ist in der Literatur häufig nicht genügend beachtet worden. So benutzt etwa Johnson ${ }^{4}$ das System (16) mit der Kontinuitätsgleichung (10), TER HAAR und Neaves ${ }^{5}$ (15) mit (10). Dies hat im letzteren Fall z. B. zur Folge, daß in der Gleichung für die Wärmetönung neben der Jouleschen Wärme, der Thomsonschen Wärme und der Wärmeleitung noch ein Glied $-\mathfrak{i} \cdot \operatorname{grad}\left(\zeta_{0} / e\right)$ auftritt, das keinen physikalischen Inhalt besitzt.

\footnotetext{
4 V. A. Johnson, Progress in Semiconductors, Vol. I, S. 63, Heywood and Comp. Ltd., London 1956.

5 D. ter Haar u. A. Neaves, Adv. Phys. 5, 241 [1956].
} 
Mit Hilfe einer der vier möglichen Gleichungen des Typs (6) und einer der Gln. (9) bis (12) lassen sich dann die spezifische Leitfähigkeit und die verschiedenen thermoelektrischen Koeffizienten bestimmen. Dazu bringt man das gewählte Gleichungssystem zweckmäßig in die Form

$$
\begin{gathered}
\frac{1}{e} \operatorname{grad} \zeta=\frac{1}{\sigma} \mathfrak{i}+\varphi \operatorname{grad} T, \mathfrak{w}_{\xi}=\Pi \mathfrak{i}-\varkappa \operatorname{grad} T, \\
\frac{\partial u}{\partial t}=\frac{i^{2}}{\sigma}+\operatorname{div}(\varkappa \operatorname{grad} T)-\mu_{\mathrm{E}} \mathfrak{i} \cdot \operatorname{grad} T,
\end{gathered}
$$

wo $\sigma, \varphi, \Pi, \varkappa$ und $\mu_{\mathrm{E}}$ direkt die spez. Leitfähigkeit, die differentielle Thermospannung, den Peltier-Koeffizienten, die spez. Wärmeleitfähigkeit und den Thomson-Koeffizienten bedeuten. Die aus den OnsaGER-Beziehungen geforderte Symmetrie der Koeffizientenmatrix der Gleichungen des Typs (6) sorgt dann automatisch für die Erfüllung der ThomsonBeziehungen

$$
\mu_{\mathrm{E}}=T \frac{\partial}{\partial T} \varphi=T \frac{\partial}{\partial T}\left(\frac{\Pi}{T}\right) .
$$

\section{Explizite Bestimmung der Leitungs- koeffizienten}

Zur expliziten Bestimmung der in (17) enthaltenen Leitungskoeffizienten, d. h. zur Bestimmung der in (6) bzw. einer äquivalenten Gleichung auftretenden $L_{i k}$ wird allgemein die Boltzmannsche Stationaritätsbedingung herangezogen. Diese liefert für die gestörte Verteilungsfunktion der Elektronen im isotropen Halbleiter in erster Näherung ${ }^{6}$

$$
f=f_{0}-\frac{l}{v}\left(\mathfrak{b} \cdot \operatorname{grad}_{\mathrm{r}} f_{0}+\dot{\mathfrak{b}} \cdot \operatorname{grad}_{\mathrm{v}} f_{0}\right),
$$

wo $f$ die Verteilungsfunktion im Lagen-Geschwindigkeits-Raum (Anzahl der Elektronen im Volumenelement $\left(\mathfrak{r}, \mathrm{d}^{3} r, \mathfrak{b}, \mathrm{d}^{3} v\right), l$ die freie Weglänge und $f_{0}$ die ungestörte Verteilungsfunktion (Zustandsdichte mal Fermi-Verteilung)

$$
f_{0}=2\left(\frac{m^{*}}{h}\right)^{3}\left(1+\exp \left\{\frac{E-\zeta}{k T}\right\}\right)^{-1}
$$

bedeuten. In (19) ist die Gradientenbildung im ersten Glied rechts bei konstantem $\mathfrak{b}$, im zweiten Glied bei konstantem $\mathfrak{r}$ vorzunehmen. Da in $E$ nur der Anteil $E_{\mathrm{n}}=E-E_{\mathrm{L}}$ von $\mathfrak{b}$ abhängt $\left(E_{\mathrm{n}}=m^{*} v^{2} / 2\right)$, setzt man in (20) zweckmäßig $E-\zeta=E_{\mathrm{n}}-\zeta_{\mathrm{n}}$ und

6 vgl. etwa O. Madelung, Halbleiter, in Handbuch der Physik, Bd. 20, Springer-Verlag, Berlin-Göttingen-Heidelberg 1957. erhält

$$
\begin{aligned}
& \operatorname{grad}_{\mathrm{r}} f_{0}=-\frac{\partial f_{0}}{\partial E_{\mathrm{n}}}\left(\frac{E_{\mathrm{n}}-\zeta_{\mathrm{n}}}{T} \operatorname{grad} T-\operatorname{grad} \zeta_{\mathrm{n}}\right), \\
& \operatorname{grad}_{\mathrm{v}} f_{0}=\frac{\partial f_{0}}{\partial E_{\mathrm{n}}} m^{*} \mathfrak{v} .
\end{aligned}
$$

Für $\dot{\mathfrak{b}}$ wird in der Literatur allgemein $-\left(e / m^{*}\right) \mathfrak{F}$ angesetzt. Dies ist jedoch inkorrekt. Die auf das Flektron wirkende „äußere“ Kraft $\Re=m^{*} \dot{\mathfrak{b}}$ ist nicht allein der Gradient des elektrostatischen Potentials, sondern der Gradient der gesamten potentiellen Energie des Elektrons. Diese ist aber gleich $E-E_{\mathrm{n}}=E_{\mathrm{L}}$. Im isothermen Fall ist diese Unterscheidung gegenstandslos, da dann $\operatorname{grad} E_{\mathrm{L}}=\operatorname{grad}(-e \psi)$ ist. Bei Gegenwart eines Temperaturgradienten ist dagegen die Temperaturabhängigkeit von $E_{\mathrm{L}}$ mit zu berücksichtigen. Man hat also zu setzen:

$\dot{\mathfrak{v}}=-\frac{1}{m^{*}} \operatorname{grad} E_{\mathrm{L}}=-\frac{e}{m^{*}} \mathfrak{E}-\frac{1}{m^{*}} \frac{\partial E_{\mathrm{L} 0}}{\partial T} \operatorname{grad} T$.

Hiermit wird (19) :

$f=f_{0}+\frac{l}{v} \frac{\partial f_{0}}{\partial E_{\mathrm{n}}} T \mathfrak{v} \cdot\left(\frac{1}{T} \operatorname{grad} \zeta-\left(E_{\mathrm{n}}-\zeta_{\mathrm{n}}\right) \operatorname{grad} \frac{1}{T}\right)$

$$
\begin{aligned}
& =f_{0}+\frac{l}{v} \frac{\partial f_{0}}{\partial E_{\mathrm{n}}} \mathfrak{b} . \\
& \left\{e \mathfrak{E}+\left[\frac{E_{\mathrm{n}}}{T}+T \frac{\partial}{\partial T}\left(\frac{\zeta_{\mathrm{n}}}{T}\right)+\frac{\partial E_{\mathrm{L} 0}}{\partial T}\right] \operatorname{grad} T\right\} .
\end{aligned}
$$

Durch Einsetzen in die Gleichungen für den elektronischen Anteil der Stromdichten (unter Vernachlässigung aller phonon-drag-Effekte und der Gitterwärmeleitung)

$$
\begin{aligned}
\dot{\mathrm{i}} & =\int \mathfrak{b} f d^{3} v, \\
\mathfrak{m} & =\int E \mathfrak{b} f d^{3} v, \\
\mathfrak{m}_{i} & =\int E_{i} \mathfrak{v} f d^{3} v \quad(i=0, \zeta, \mathrm{n})
\end{aligned}
$$

folgen dann Gleichungen vom Typ (6), z. B. bei Verwendung von (24) in $\dot{j}$ und $\mathfrak{m}_{\zeta}$ direkt das Gleichungssystem (15) und damit die gesuchten Koeffizienten.

\section{Diskussion}

Trotz der oben erwähnten Inkonsequenz bei der Wahl der richtigen Kraft in der Boltzmansschen Stationaritätsbedingung sind die in der Literatur mitgeteilten Ergebnisse der Theorie fast durchweg richtig. Dies hat verschiedene Gründe.

So wird in vielen (vor allem frühen) Arbeiten über die thermoelektrischen Effekte in Halbleitern 
als Energienullpunkt die Bandkante $E_{\mathrm{L}}$ selbst gewählt. Dies hat zur Folge, daß auf der rechten Seite von (23) das Glied $\operatorname{grad} E_{\mathrm{L} 0}$ fehlt, also

$$
\dot{\mathfrak{v}}=-\frac{e}{m^{*}} \mathfrak{F}
$$

wird. Außerdem wird dann $\mathfrak{w}=\mathfrak{w}_{\mathrm{n}}, E=E_{\mathrm{n}}$ und $\zeta=\zeta_{\mathrm{n}}$. Alle $E_{\mathrm{L}}$ enthaltenden Glieder der oben angegebenen Gleichungen fallen fort und diese Vernachlässigungen heben sich gerade bei der Berechnung der thermoelektrischen Effekte eines Störstellenleiters heraus. Berechnet man jedoch auf diese Weise die Beiträge der beiden Bänder getrennt und kombiniert sie in der richtigen Weise, so liefern die Ergebnisse bekanntlich nicht die von der ambipolaren Diffu$\operatorname{sion}^{7}$ herrührenden Beiträge. Nur in diesen Beiträgen macht sich die Temperaturabhängigkeit der Bandkanten, also der Breite der verbotenen Zone, bemerkbar.

Andererseits wird häufig in (23) und damit in (24 a) das Glied $\operatorname{grad} E_{\mathrm{L} 0}$ unberücksichtigt gelassen, das System (16) angewandt, für die Energiebilanz jedoch (10) benützt. Berechnet man dann etwa die Thermospannung aus dem THomson-Koeffizienten, so heben sich beide Fehler gerade wieder heraus.

Auch bei Berücksichtigung der Temperaturabhängigkeit der Bandkanten, aber Ersetzen von $m^{*} \dot{\mathfrak{y}}$ durch $-e$ F kommt man zu richtigen Ergebnissen, wenn man in (19) $\operatorname{grad}_{\mathrm{r}} f_{0}$ nicht bei festem $E_{\mathrm{n}}$, sondern festem $E$ bildet. Auf diese inkorrekte Weise kam u. a. der Verfasser in einer früheren Arbeit ${ }^{6}$ zur richtigen Verteilungsfunktion (24a) ${ }^{8}$.

In vielen Fällen ist die Temperaturabhängigkeit der Bandkanten so klein, daß ihre Vernachlässigung

7 P. J. Price, Phil. Mag. 46, 1252 [1955].

8 Wir brauchen deshalb hier nicht auf die Ergebnisse der Theorie einzugehen und können für die explizite Angabe der Gleichungen für die thermoelektrischen Koeffizienten auf 1. c. ${ }^{6}$ verweisen. keinen großen Fehler bringt. Dies ist jedoch nicht immer so. Bei der Berechnung des ambipolaren Diffusionsstromes im nicht-isothermen gemischten Halbleiter ${ }^{7}$ ergibt sich beispielsweise eine Proportionalität dieses Stromes zu $(\Delta E+4 k T)$. Ohne Berücksichtigung der Temperaturabhängigkeit der Bandkanten ist hier statt $\Delta E(T)$ nur $\Delta E(0)$ zu setzen. Etwa im Falle des InSb reduziert sich hierdurch bei Zimmertemperatur dieser Faktor um fast 30 Prozent ${ }^{9}$.

Ein weiterer Fall, in welchem der Ansatz

$$
\dot{\mathfrak{v}}=-\frac{e}{m^{*}} \mathfrak{F}
$$

versagt, findet sich in der Theorie der inhomogenen Halbleiter. Liegt die Inhomogenität nicht nur in einer ortsabhängigen Störstellenverteilung, sondern in einer variablen Bandstruktur (Mischkristalle örtlich variabler Zusammensetzung), so ist auch $m^{*}$ und somit der „kinetische“ Anteil der Energie ortsabhängig. In diesem Fall ist

$$
\dot{\mathfrak{b}}=-\frac{e}{m^{*}} \operatorname{grad} E
$$

zu setzen. Man erhält dann für die gestörte Verteilungsfunktion wieder Gl. (24), jetzt aber mit ortsabhängiger freier Weglänge $l$. Auch die phänomenologischen Gln. (17) bleiben erhalten, nur werden jetzt die Koeffizienten $\sigma, \varphi, \Pi, \varkappa$ und $\mu_{\mathrm{E}}$ Variable des Ortes.

Herrn Prof. Dr. W. Sснотткy danke ich für eine lehrreiche Diskussion.

9 Price vernachlässigt in seiner Arbeit ${ }^{7}$ die Temperaturabhängigkeit von $\triangle E$. Bei Berücksichtigung dieses Einflusses ist in seiner Gl. (11) zwar $\Delta=\Delta E(T)$ zu setzen, in (10) aber $\Delta=\Delta E(0)\left[n_{1} n_{2} \sim \exp (-\Delta E(0) / k T)\right]$. Gleichzeitig tritt dann aber in seiner Gl. (2) ein zusätzliches Glied $\left(\mu_{i} / e\right) \operatorname{grad} E_{i}\left(E_{i}=E_{\mathrm{L}}\right.$ bzw. $\left.-E_{\mathrm{V}}\right)$ auf, so daß im Endresultat der Gl. (12) wieder $\Delta=\Delta E(T)$ ist. Vgl. hierzu l. c. ${ }^{6}$, Gln. (35.3) und (36.4). 